\title{
Sistema de suministro de energía eléctrica para actuadores basados en aleaciones con memoria de forma
}

\author{
Electric energy supply system for actuators based on shape memory alloys
}

\author{
Luis A. Mier-Quiroga ${ }^{1 *} \quad$ César L. Ramírez-Chávez ${ }^{1} \quad$ Daniel Maldonado-Onofre ${ }^{1}$
}

Recibido 21 de noviembre de 2017, aceptado 23 de diciembre de 2018

Received: November 21, 2017 Accepted: December 23, 2018

\begin{abstract}
RESUMEN
Las aleaciones con memoria de forma son metales que pertenecen a una clase de materiales con el mismo tipo de memoria. Este tipo de materiales tienen la habilidad de recordar su estado previo cuando son sometidos a ciertos estímulos tales como variaciones eléctricas, magnéticas y térmicas. Las aleaciones de metales que tienen esta característica también son conocidas como "alambres musculares", debido a que su principal objetivo es generar movimiento sin el uso de motores o solenoides. Estudios publicados han demostrado que, en el caso de los alambres musculares, un sobrecalentamiento del material debido a un exceso de corriente eléctrica a través de él, puede mermar su confiabilidad y durabilidad. En este artículo se presenta el diseño de un sistema de alimentación de energía eléctrica para alambres musculares, basado en el convertidor buck. El sistema incluye una etapa de control basado en lógica difusa capaz de regular la corriente que circula a través del alambre muscular. Los resultados obtenidos en las simulaciones del sistema que se presenta muestran que la corriente es ajustada a un valor máximo, establecido por el fabricante del alambre, ante diversas perturbaciones.
\end{abstract}

Palabras clave: Alambre muscular, convertidor buck, control difuso.

\begin{abstract}
The shape memory alloys are metals that belong to a class of shape-memory materials. These kinds of materials can memorize their previous form when they are subject to stimuli such as electric, magnetic, and thermic variations. The metal alloys that exhibit this characteristic are commonly named "muscle wires" because their main objective is to generate motion without electric motors or coils. Published studies have demonstrated that, in the case of the muscle wires, their durability and reliability can decrease due a material overheating as a consequence of overcurrent. The design of a buck converter based electric energy supply system for muscle wires is presented in this paper. A control stage based on fuzzy logic is included in the system to regulate the current through the muscle wire. The electrical current adaptation to a maximum magnitude, specified by the muscle wire manufacturer, is achieved in the presence of disturbances, according to the simulation results of the proposed system.
\end{abstract}

Keywords: Muscle wire, buck converter, fuzzy control.

1 Departamento de Ingeniería Electromecánica. Tecnológico de Estudios Superiores de Jocotitlán. Jocotitlán, Estado de México, México.E-mail: luis.mier@tesjo.edu.mx; cesar.ramirez@tesjo.edu.mx; daniel.maldonado@tesjo.edu.mx

* Autor de correspondencia: luis.mier@tesjo.edu.mx 


\section{INTRODUCCIÓN}

Los alambres musculares son aleaciones de metales que pertenecen a los materiales que presentan la característica de tener "memoria de forma" es decir, tienen la capacidad de memorizar o mantener su forma previa cuando son sometidos a ciertos estímulos como variaciones magnéticas o termomagnéticas [1]. Desde el año de 1932, se ha experimentado con este tipo de materiales con el fin de obtener, principalmente, sistemas mecánicos donde el efecto de memoria de forma puede desarrollar trabajo físico [2]. A lo largo del tiempo sus campos de aplicación han sido diversos, partiendo en 1960 de la industria aeroespacial y de comunicaciones con el desarrollo de antenas satelitales que deben de expandirse o contraerse, pasando por aplicaciones domésticas en ventanas que se abren o cierran de acuerdo a la temperatura ambiente, hasta extremidades de robots humanoides o prótesis de extremidades humanas que obtienen su capacidad de movimiento gracias a los alambres musculares [2]. Recientemente se ha diversificado el campo de aplicación de actuadores desarrollados en base a este tipo de materiales gracias a su simplicidad, relación fuerza-peso, bajo costo, operación silenciosa y tiempo de vida útil relativamente amplio [2-3]. Esta última característica está directamente relacionada con el "buen manejo" del alambre muscular.

Sea cual sea la aplicación, es imperante monitorear y controlar la cantidad de energía aplicada al alambre y así evitar su sobrecalentamientos y en consecuencia, daño del actuador [1]. Existe poca o nula información, referente a la etapa de alimentación del alambre, en los trabajos que se proponen diversas aplicaciones de estos materiales. Generalmente, sólo se toma la energía de la fuente de alimentación y mediante algún dispositivo o circuito tan básico como un interruptor, basándose en el principio encendido/apagado, controlan la potencia eléctrica proporcionada al alambre. Los proyectos más sofisticados utilizan la modulación de ancho de pulso para controlar la conmutación de los dispositivos semiconductores, a través de los cuales circula la corriente que modifica la potencia eléctrica que permite la elongación o contracción de los alambres.

Con el fin de mantener la vida útil indicada por el fabricante, en este trabajo se propone una fuente de alimentación, basada en el convertidor buck, controlada mediante lógica difusa, para alimentar sistemas que utilizan alambres musculares como dispositivos acción mecánica. El control difuso tiene su origen en los sistemas basados en reglas para la toma de decisiones y en la lógica difusa para la evaluación de ellas. Es una alternativa práctica para una amplia variedad de retos en el control aplicado. Proporciona un método práctico para realizar controladores no lineales mediante el uso de información obtenida por la experiencia [4].

La organización del presente artículo es la siguiente; inicialmente se expone la teoría básica respecto a los alambres musculares, convertidores buck y controladores basados en lógica difusa. A continuación, se presenta el diseño del convertidor buck y su controlador difuso. Posteriormente, se presentan los resultados y discusiones sobre las simulaciones hechas en el programa Matlab, para analizar el comportamiento del sistema de alimentación controlado, ante diversas eventualidades que pueden presentarse en aplicaciones reales. Finalmente se exponen las conclusiones obtenidas del análisis de los resultados obtenidos.

\section{ANÁLISIS TEÓRICO}

\section{Alambres musculares}

Actualmente, la mezcla de la que se compone el alambre muscular es de níquel y titanio, con partes casi iguales de uno y otro. El más pequeño cambio en la relación entre los dos elementos tiene un efecto dramático en la temperatura de transición de la aleación resultante. Cada empresa que produce nitinol debe mantener la relación de los componentes a un exacto valor para asegurar una temperatura de transición estable y repetible [1].

Es posible hacer que la aleación recuerde una forma en particular. La aleación se puede deformar y se le puede hacer volver a la forma original calentando la aleación por encima de una temperatura llamada "de transición". La fuerza generada cuando el alambre está volviendo a su forma es considerable, una pulgada cuadrada de material de nitinol genera una fuerza de retorno a la forma de 30 PSI [2]. Esta característica física tan especial del nitinol se basa en su estructura cristalina dinámica y sensible al calor. Cuando el nitinol está deformado, la estructura cristalina no se destruye, sino que se transforma, 
cambiando a una singular disposición cristalina. En los metales normales, en cambio, las deformaciones hacen que la estructura molecular se deshaga, dejando a los átomos en nuevas posiciones cristalinas. A causa de esto, el cristal no puede conservar una "memoria" de dónde estaban los átomos antes de moverse. El movimiento físico del nitinol se debe a la reestructuración interna de las moléculas. Y como el movimiento se genera a nivel molecular, es muy potente [1].

El alambre de nitinol tiene una resistencia eléctrica alta (para ser un conductor metálico), de aproximadamente $0,5 \mathrm{ohms}$ por centímetro en el alambre de 6 milésimas de pulgada. La resistencia del alambre a la corriente eléctrica produce con rapidez un calor suficiente como para llevar al alambre a su temperatura de transición [1].

Usualmente se aplica una fuerza de tensión opuesta a la dirección de contracción del alambre de nitinol. Esta fuerza repone al alambre a su longitud original en la fase de baja temperatura. A esta fuerza aplicada se le llama fuerza de extensión. Si se lleva un alambre de nitinol a su temperatura de transición sin una fuerza de extensión, se contraerá, pero cuando se enfríe no retornará a su longitud original. El tiempo de reacción puede ser reducido, en el orden de los milisegundos utilizando pulsos de corriente elevada. Cuanto más rápido se mueva una determinada masa, más grande será la inercia a vencer. Si la inercia es mayor a la resistencia a la tracción del alambre, este se cortará [5]. En la respuesta al calor del nitinol se observa una curva de histéresis. Cuando sube la temperatura, la relación de temperaturacontracción es más rápida, al descender, a las mismas temperaturas corresponde un relajamiento menor al que se podría esperar si la curva fuese lineal. Este efecto de retraso, producido por la viscosidad o fricción interna de la estructura cristalina, se puede observar en la Figura 1 [6].

\section{Convertidor Buck}

Un convertidor buck produce un voltaje promedio de salida menor al voltaje de cd de entrada, su circuito básico es como el que se muestra en la Figura 2.

Cuando el interruptor se encuentra cerrado durante el tiempo $t_{o n}$, el voltaje de entrada $V d$ aparece en los extremos del diodo y por lo tanto en un extremo del inductor. Cuando el interruptor es abierto, durante

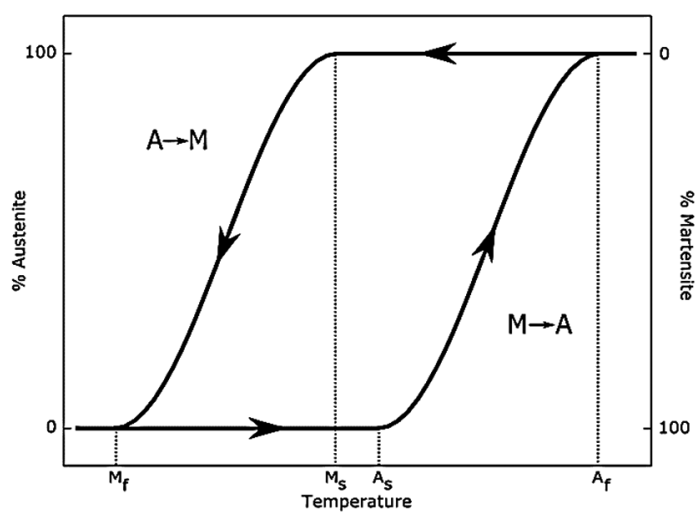

Figura 1. Fases debido al cambio de temperatura.

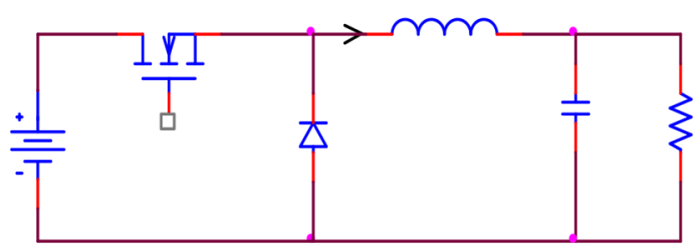

Figura 2. Convertidor buck.

el tiempo $t_{\text {off }}$, el voltaje a través del diodo puede considerarse cero ya que este se encuentra polarizado directamente debido a que la corriente almacenada por el inductor fluye a través de éste, teniendo una forma de onda de la corriente en el inductor como se muestra en la Figura 3 [7].

El voltaje de salida promedio Vo, puede ser determinado de la siguiente forma:

$$
V o=\frac{1}{T s} \int_{0}^{T s} V o(t) d t=\frac{1}{T s} \int_{0}^{t_{\text {on }}} V d d t=\frac{V d}{T s} t_{\text {on }}
$$

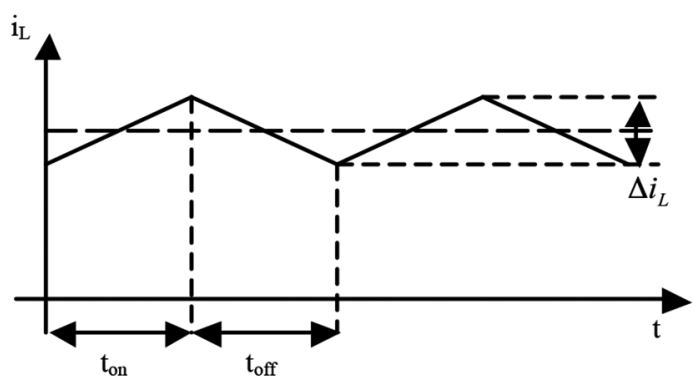

Figura 3. Forma de onda de la corriente en el inductor. 
Si se define al ciclo de trabajo,

$$
D=\frac{t_{\text {on }}}{T s}
$$

entonces, la ecuación 1 queda de la siguiente forma:

$$
V o=D V d
$$

donde $T s$ es el periodo de conmutación.

La ecuación anterior nos indica que el voltaje de salida para el modo continuo depende solamente del ciclo de trabajo [7].

El convertidor puede operar en modo continuo o discontinuo, dependiendo del comportamiento de la corriente a través del inductor $L$ [7]. Para el análisis del convertidor se asumen dos estados por periodo de conmutación Ts, en el estado de encendido $\left(t_{o n}\right)$, el transistor conduce la corriente de la fuente de entrada a través del inductor hasta la carga y en el estado de apagado $\left(t_{\text {off }}\right)$, cuando el transistor deja de conducir, la corriente del inductor fluye a través del diodo.

\section{El control difuso}

A mediados de la década de 1960, el Profesor Lotfi Zadeh, de la Universidad de California en Berkeley, introdujo su teoría de los conjuntos difusos, donde la idea de pertenencia o de no pertenencia de un elemento a un determinado conjunto fue sustituida por el grado de pertenencia de un elemento a uno o varios conjuntos. Basándose sobre esta idea, se desarrolló toda la teoría de conjuntos, redefiniendo operaciones y propiedades [8].

En el diseño de un controlador, se establecen un conjunto de reglas sobre el control del proceso. Las reglas pueden ser establecidas mediante las decisiones tomadas y acciones realizadas por el operador que controla el proceso. También, la base de reglas puede ser construida a partir de la experiencia de un ingeniero en control que ha realizado el modelo matemático, un amplio análisis y algoritmos de control del proceso a controlar. Tal conocimiento es cargado en el controlador difuso para automatizar el proceso en cuestión (Figura 4). El control difuso proporciona un método para representar e implementar las ideas sobre cómo lograr un control de alto desempeño.

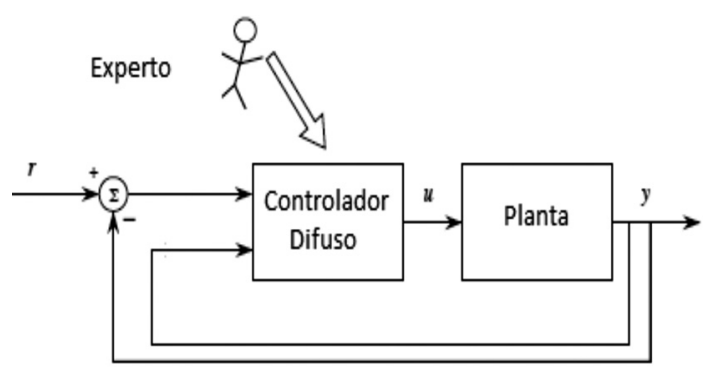

Figura 4. Sistema de control difuso.

El control difuso es una de las técnicas más utilizadas cuando no se tiene el conocimiento exacto del sistema que se desea controlar. Al igual que los "controladores clásicos", toman los valores de las variables de entrada, realizan algún procedimiento con ellas y deciden como modificar las variables de salida, afectando estas últimas a la planta. La diferencia esencial es que no procesan ecuaciones ni tablas, procesan reglas para decidir como cambiar las salidas [4].

Un sistema de control difuso está compuesto de los siguientes elementos [4].

- $\quad$ Base de reglas (conjunto de reglas If-Then). Contiene una cuantificación lógica difusa de la descripción lingüística del operador experto sobre cómo lograr un buen control.

- Mecanismo de inferencia (también llamado máquina de inferencia o módulo de inferencia difusa). Emula la decisión del experto. Interpreta y aplica el conocimiento adquirido sobre cómo realizar el mejor control de la planta.

- Interface de difusificación. Convierte las entradas del controlador en información que el mecanismo de inferencia puede usar fácilmente para activar y aplicar reglas.

- Interface de desdifusificación. Convierte las conclusiones del mecanismo de inferencia en las correspondientes entradas del proceso.

\section{Sistema de suministro de energía propuesto}

El sistema de alimentación propuesto se muestra en la Figura 5. El circuito se basa en un convertidor buck trabajando en modo de conducción continuo. La resistencia de carga del convertidor es el alambre muscular que consta de su propio sistema de accionamiento. En gran parte de las aplicaciones, el sistema de accionamiento del alambre muscular consta de un interruptor semiconductor (bjt, mosfet 
o igbt), conmutado mediante una señal modulada en ancho de pulso (PWM). El ancho del pulso que se aplica al interruptor permite controlar el porcentaje de contracción que tendrá el alambre muscular, por lo que, una señal PWM de conmutación con ciclo de trabajo del $50 \%$ contraerá en menor proporción al alambre que una señal PWM el máximo ancho de pulso $(\mathrm{D}=100 \%)$. El convertidor buck, será conmutado acorde a una señal PWM diferente a la que controla el porcentaje de contracción del alambre muscular.

El ancho de esta señal determina el ciclo de trabajo y por lo tanto, el voltaje y la corriente de salida del convertidor, es decir la potencia en el alambre muscular. Como se puede observar en la Figura 5, el sistema carece de etapa de control, por lo que al modificar alguna característica del convertidor o alambre muscular, la corriente a través de éste se verá afectada a tal grado que puede llegar a valores por encima del valor máximo que asegure un funcionamiento adecuado del alambre.

\section{Control del convertidor buck}

Debido a que el sistema que se presentó anteriormente, bajo ciertas circunstancias, puede exceder el valor de la corriente que garantiza un adecuado funcionamiento del alambre y por lo tanto su degradación y reducción de su vida útil se incorporó un sistema de control basado en lógica difusa o controlador difuso, como se muestra en la Figura 6.

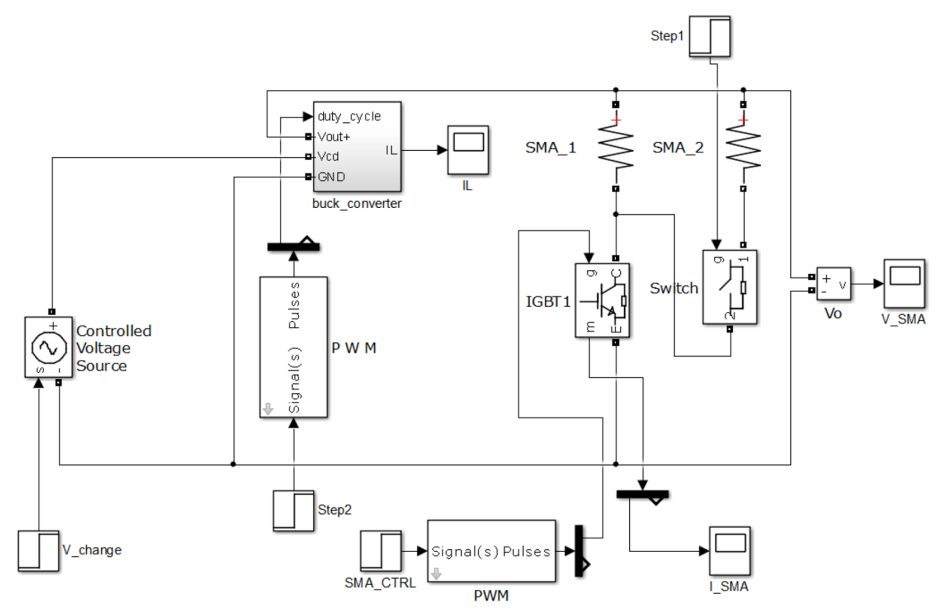

Figura 5. Sistema de suministro.

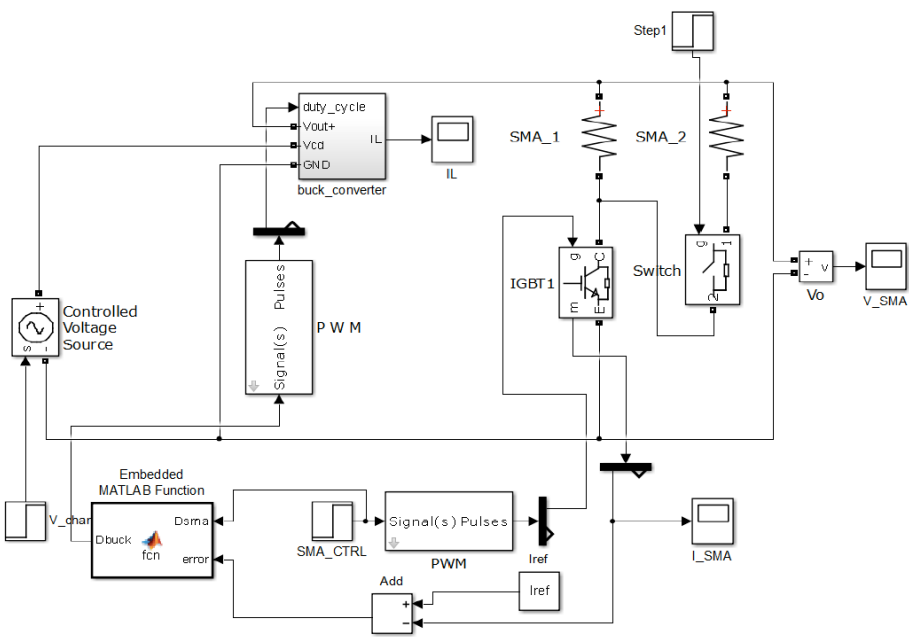

Figura 6. Sistema de suministro controlado. 
Es posible observar en la figura anterior que la entrada del sistema de control (bloque Embedded MATLAB Function), está compuesta por las variables "Dsma" y "Error". La primer entrada es para considerar el ciclo de trabajo del tren de pulsos que emite el PWM que controla la contracción del alambre muscular. El error permite conocer que tan alejada está la corriente a través del alambre, con respecto a la de referencia, que es la que garantiza su adecuado manejo.

La Figura 7 muestra las funciones de pertenencia de las variables de entrada del controlador difuso.

La variable de salida del controlador se llama "Dbuck", sus funciones de pertenencia se muestran en la Figura 8. Este valor de salida es el ancho de pulso que debe tener el PWM que conmuta el interruptor del convertidor buck.

El ancho de pulso entregado por el controlador, garantiza que la corriente que circula a través del alambre muscular no exceda el valor que asegura un adecuado funcionamiento.

En la Tabla 1, se muestra la base de reglas del sistema de control difuso y se proponen acorde a las acciones que debe de tomar distintos casos presentes en las variables de entrada.

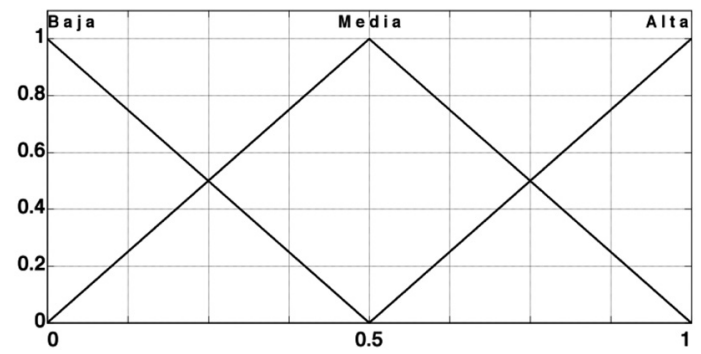

(a) Dsma.

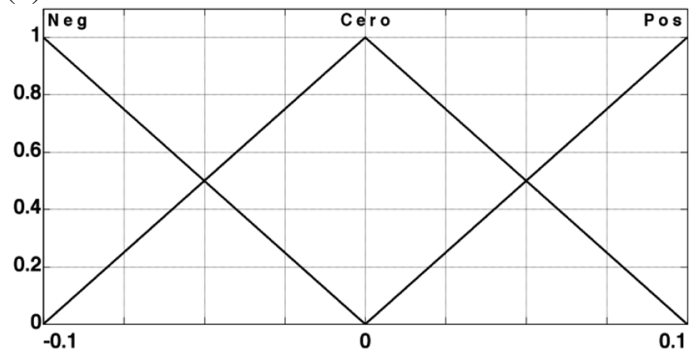

(b) Error.

Figura 7. Funciones de pertenencia de las variables de entrada.

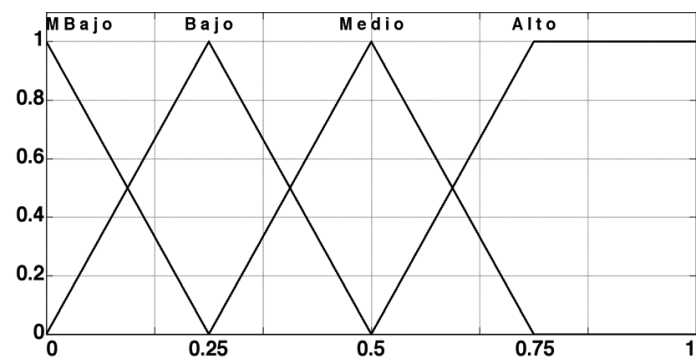

Figura 8. Funciones de pertenencia de la variable de salida, Dbuck.

Tabla 1. Base de reglas del controlador difuso.

\begin{tabular}{|c|c|c|c|c|}
\hline \multirow{2}{*}{ Dbuck } & \multicolumn{3}{c|}{ Dsma } \\
\cline { 3 - 5 } \multicolumn{2}{|c|}{} & Baja & Media & Alta \\
\hline \multirow{3}{*}{ Error } & Neg & MBajo & MBajo & Bajo \\
\cline { 2 - 5 } & Cero & Bajo & Medio & Alto \\
\cline { 2 - 5 } & Pos & Bajo & Medio & Alto \\
\hline
\end{tabular}

\section{RESULTADOS}

\section{Simulación}

Se simuló, tanto el sistema de suministro de energía sin control, como el que cuenta con la etapa de control difuso. En ambos casos se propusieron dos perturbaciones, la primera consiste en, después de un determinado tiempo, agregar una resistencia en paralelo con el alambre muscular de tal forma que se simule la reducción en resistencia que presenta el alambre al contraerse y la segunda perturbación consiste en modificar el voltaje de entrada del convertidor, incrementándolo en un $50 \%$. Se diseñaron tres convertidores buck, cada uno para ciertas características eléctricas de alambres musculares comerciales, las características del controlador difuso son iguales en cada caso.

La Figura 9 muestra la respuesta en corriente en el alambre muscular y voltaje del convertidor buck. Se analizó el caso de un alambre muscular con diámetro de $375 \mu \mathrm{m}$ y resistencia lineal de 8 $\Omega / \mathrm{m}$. En este caso el fabricante recomienda una corriente máxima de 2,75 A. Para las respuestas que se mostrarán a continuación, las gráficas en color negro corresponden al sistema en lazo abierto y las de color rojo en lazo cerrado. La Figura 10 muestra la respuesta en corriente y voltaje, proporcionados por el sistema para un 
(a) Corriente.

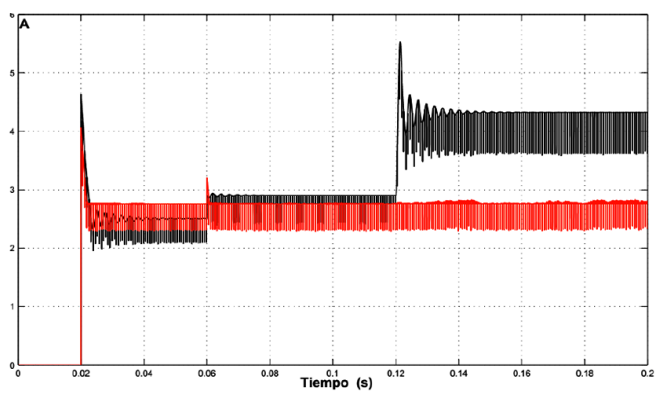

(b) Voltaje.

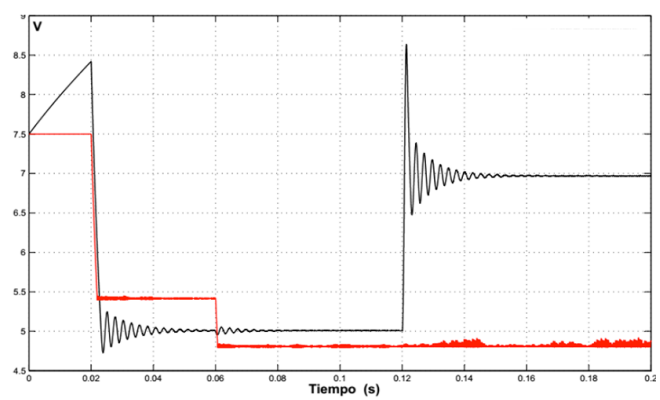

Figura 9. Respuesta del sistema con y sin etapa de control para un alambre de $375 \mu \mathrm{m}$.

(a) Corriente.

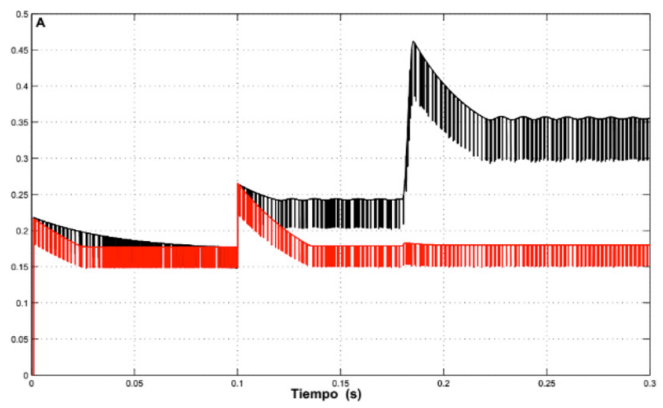

(b) Voltaje.

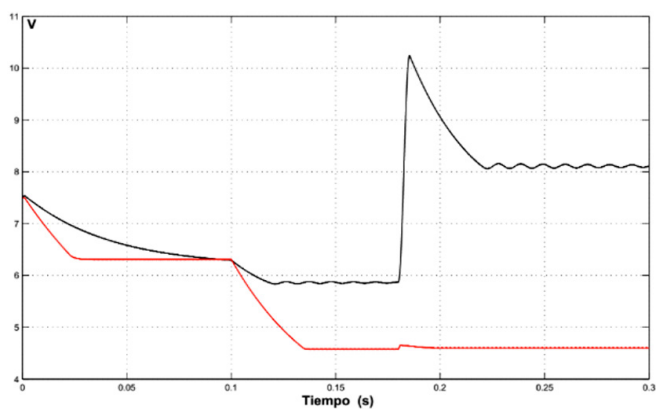

Figura 10. Respuesta del sistema con y sin etapa de control para un alambre de $100 \mu \mathrm{m}$. alambre muscular de $100 \mu \mathrm{m}$ de diámetro y resistencia lineal de $150 \Omega / \mathrm{m}$, con una corriente máxima recomendada de 0.170 A. En la Figura 11 se muestra la respuesta en corriente y voltaje para el caso de un alambre muscular de $150 \mu \mathrm{m}$ de diámetro y resistencia lineal de $50 \Omega / \mathrm{m}$, con una corriente máxima recomendada de $0,4 \mathrm{~A}$.

En las figuras anteriores es posible observar que, en el momento en que inicia el sistema de suministro de energía, al momento de contraerse el alambre (instante en que disminuye la resistencia de carga del convertidor) y cuando se aumenta del voltaje de entrada, las respuestas de corriente presentan sobreimpulso y oscilaciones. En el caso del convertidor que cuenta con el control difuso, sus magnitudes se estabilizan en el valor de referencia y prácticamente desaparecen las oscilaciones, lo que no ocurre en el caso del convertidor sin control. Para el caso de las señales que corresponden al voltaje del controlador, es posible observar el ajuste en su magnitud, debido a la acción de control, para mantener la referencia, acción que no sucede en el caso del sistema de suministro sin control.

(a) Corriente.

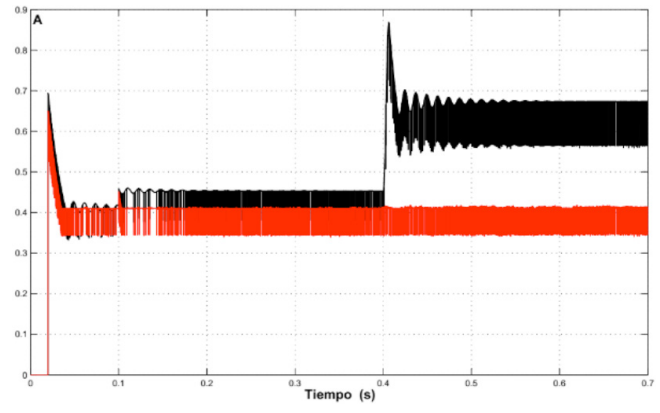

(b) Voltaje.

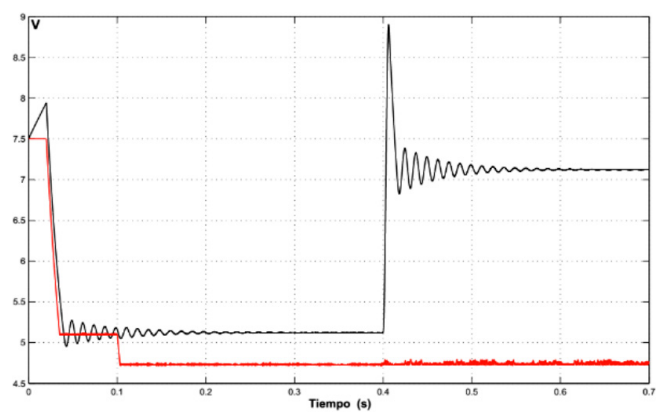

Figura 11. Respuesta del sistema con y sin etapa de control para un alambre de $150 \mu \mathrm{m}$. 


\section{Caso experimental}

Para probar el sistema de suministro de energía con control, se armó el circuito mostrado por bloques en la Figura 12, para el caso del alambre muscular mostrado en la Figura 13 (diámetro de $375 \mu \mathrm{m}$ y resistencia lineal de $8 \Omega / \mathrm{m}$ ).

En la Figura 14 se puede ver el circuito real, que se basa en una tarjeta de desarrollo del fabricante Cypress, que cuenta con un microcontrolador PSoC 5.

El PSoC 5 se programó con el algoritmo de control difuso que tiene las características que fueron descritas antes en la sección correspondiente. En el mismo microcontrolador se configuraron dos módulos PWM. El primer módulo PWM genera una señal de $20 \mathrm{kHz}$, la cual conmuta el transistor MOSFET que ajusta la contracción del alambre muscular. El segundo módulo PWM, genera una señal de 50 kHz y conmuta al MOSFET del convertidor buck.

El voltaje de salida del convertidor buck varía acorde al valor del ciclo de trabajo determinado por el controlador difuso (variable de salida Dbuck). En el mismo programa del microcontrolador se ajusta el valor de referencia del sistema de control, así como el ciclo de trabajo referente al alambre muscular. El PSoC 5 cuenta internamente con 4 convertidores analógico-digital, de los cuales se configuró uno para procesar el voltaje generado por el sensor ACS712ELCTR-05B-T, que es proporcional a la corriente que circula en el alambre muscular. Para probar el sistema de energía controlado se realizó un experimento similar al caso de la simulación. $\mathrm{Al}$ encender el sistema se genera una señal para el primer PWM con un ciclo de trabajo del $0 \%$ que,

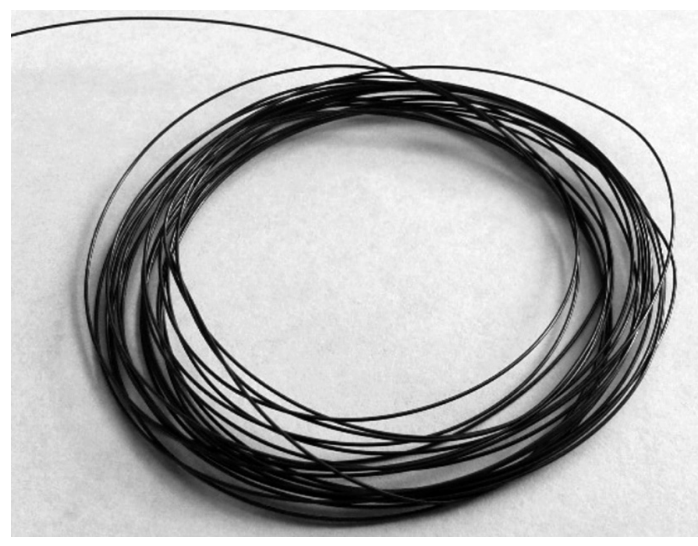

Figura 13. Alambre muscular.

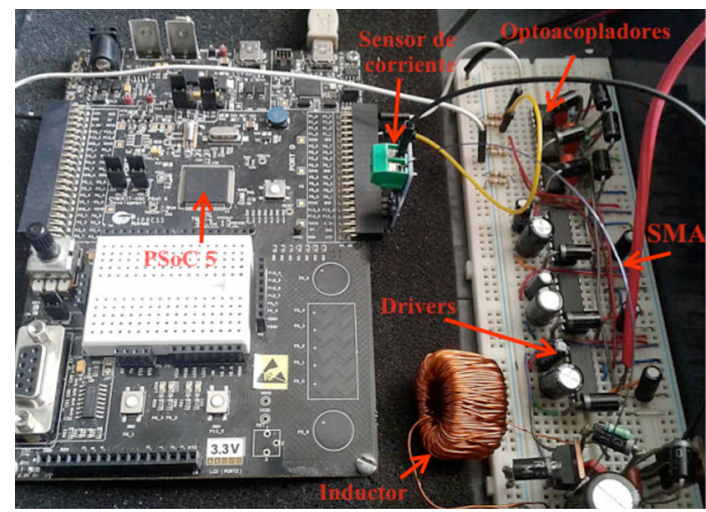

Figura 14. Circuito experimental.

después de medio segundo segundo, es incrementado al 100\%. Posteriormente, después de cuatro y medio segundos, aunados a los dos anteriores, se incrementa el voltaje de alimentación del convertidor buck. La

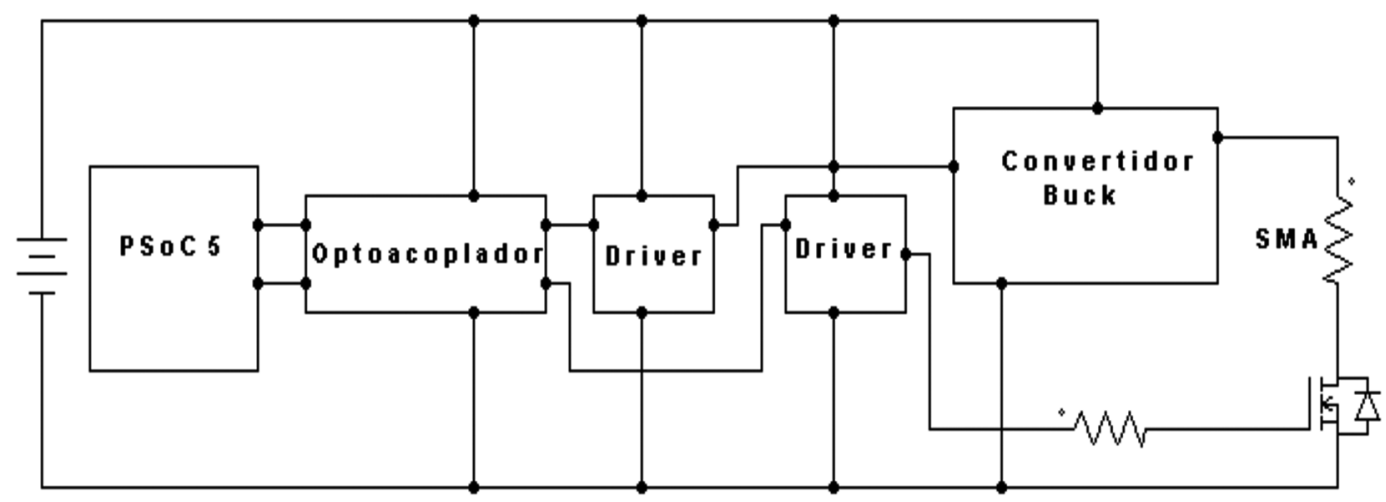

Figura 12. Diagrama por bloques del sistema de suministro de energía. 


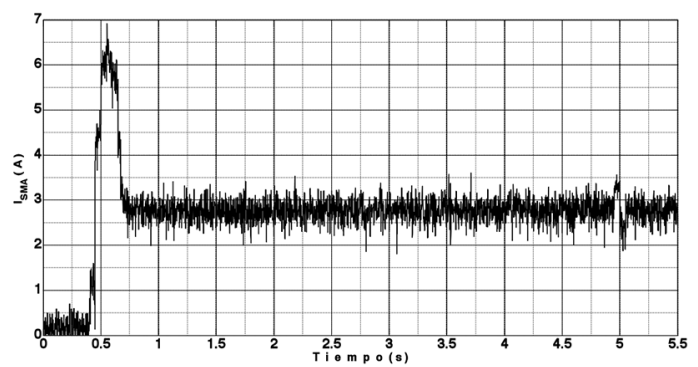

Figura 15. Respuesta de corriente a través del alambre muscular.

Figura 15 muestra la respuesta de la corriente que circula a través del alambre muscular.

La respuesta de la corriente mostrada en la figura anterior, permite corroborar que el controlador ajusta el ciclo de trabajo del convertidor buck, de tal forma que la corriente en el alambre muscular se mantenga en el valor de referencia, aún con la presencia de perturbaciones.

\section{CONCLUSIONES}

Se diseñó un sistema de suministro de energía eléctrica controlada, basado en el convertidor buck, para actuadores accionados por alambres musculares. El sistema cuenta con la característica de controlar la corriente de circula a través del alambre. El sistema de control, basado en lógica difusa, ajusta el voltaje proporcionado por el convertidor al alambre de tal forma que ante perturbaciones, exista la mínima variación en la corriente del alambre, respecto a una corriente de referencia. Se corroboró el correcto funcionamiento del sistema propuesto mediante simulaciones y de forma experimental. Los resultados indican que un sistema con estas características, permite operar a los alambres musculares de forma adecuada, asegurándose que la máxima corriente de trabajo permitida no se exceda, garantizando la vida útil estipulada por el fabricante del alambre.

\section{REFERENCIAS}

[1] J.M. Jani, M. Leary, A. Subic and M.A. Gibson. "A review of shape memory alloy research, applications and opportunities". Materials \& Design. Vol. 56, pp. 1078-1113. 2014. ISSN: 0264-1275. DOI: 10.1016/j. matdes.2013. 11.084.

[2] R.G. Gilberston. "Muscle Wires Project Book". Mondo Tronics Inc. 3rd Edition. San Rafael CA, EUA, pp. 2-11. 2005. ISBN: 1-879896-13-3.

[3] H. Kim, Y. Han, D.Y. Lee, J.I. Ha and K.J. Cho. "Sensorless displacement estimation of a shape memory alloy coil spring actuator using inductance". Smart Materials and Structures. Vol. $22 \mathrm{~N}^{\circ}$ 2. 2013. DOI: 10.1088/0964-1726/22/2/025001.

[4] K.M. Passino and S. Yurkovich. "Fuzzy control". Addison-Wesley. Menlo Park, CA, EUA, pp. 23-77. 1998. ISBN: 020118074X, 9780201180749.

[5] S.K. Chaitanya and K. Dhanalakshmi. "Control of shape memory alloy actuated gripper using Sliding Mode Control”. IEEE International Conference on Control Applications, pp. 1247-1252. 2013. ISSN: 1085-1992. DOI: 10.1109/CCA.2013. 6662923.

[6] I.M. Dynalloy. "Technical Characteristics of Flexinol Actuator Wires". DYNALLOY. 2010.

[7] N. Mohan, T.M. Undeland and W.P. Robbins. "Power electronics: converters, applications, and design". John Wiley \& Sons Inc. 3rd Edition. EUA, pp. 232-378. 2002. ISBN: 978-0471226932.

[8] T.J. Ross. "Fuzzy logic with engineering applications". John Wiley \& Sons Inc. $3^{\text {rd }}$ Edition. EUA, pp. 25-42. 2010. ISBN: 978-0-470-74376-8. 